\title{
SOME PHENOMENA IN HOMOTOPICAL ALGEBRA
}

\author{
K. VARADARAJAN
}

\begin{abstract}
In [6] D. G. Quillen developed homotopy theory in categories satisfying certain axioms. He showed that many results in classical homotopy theory (of topological spaces) go through in his axiomatic set-up. The duality observed by Eckmann-Hilton in classical homotopy theory is reflected in the axioms of a model category. In [7] we developed the theory of numerical invariants like the Lusternik-Schnirelmann category and cocategory etc. for such model categories and in [8] we dealt with applications of this theory to injective and projective homotopy theory of modules as developed by Hilton [2], [3, Chapter 13].

Contrary to the general expectations there are many aspects of classical homotopy theory which cannot be carried over to Quillen's axiomatic set-up. This paper deals with some of these phenomena.
\end{abstract}

Introduction. For any topological group $G$ it is well known [1], [5], that there exists a principal fibre space $E_{G} \rightarrow{ }^{n_{G}} B_{G}$ with group $G$ and total space $E_{G}$ contractible. This suggests the following question. Suppose $M$ is a group object in a model category $\mathscr{C}$ in the sense of Quillen [6]. Does there exist a fibration $E \rightarrow{ }^{p} B$ in $\mathscr{C}$ with the property that $E$ is contractible (i.e. to say $\pi(R Q(E), R Q(E))=0$ following the notation of Quillen [6]) with fibre of $p$ isomorphic to $M$ ? We will give examples to show that, in general, this is false. Also we will illustrate that, given a cogroup object $H$ in a model category $\mathscr{C}$, there need not exist a cofibration $A \rightarrow^{q} E$ in $\mathscr{C}$ with $E$ contractible and cofibre of $q$ isomorphic to $H$.

Actually it will turn out that the two model categories $\mathscr{C}$ and $\mathscr{F}$ that we mention in this connection $(\S 1)$ will have the following additional properties.

(i) All the objects are simultaneously group objects and cogroup objects.

(ii) For every object $A$ both $\Sigma A$ and $\Omega A$ are contractible.

It can easily be shown that in the category $\mathscr{T}$ of topological spaces if $G$ is a group object with $\Sigma G$ contractible then $G$ itself is contractible.

In $\S 2$ we characterise all $\mathrm{CW}$-complexes $X$ with the property that $\Sigma X$ is contractible. They turn out to be "Moore CW-complexes" $M(\pi, 1)$

Received by the editors June 25, 1973.

AMS (MOS) subject classifications (1970). Primary 18G05, 55D30, 55D35, 55D40, $55 \mathrm{D} 50$.

Key words and phrases. Category, cocategory, $p$-homotopy, $i$-homotopy, Moore spaces.

(c) American Mathematical Society 1974 
for groups $\pi$ satisfying $H_{1}(\pi)=0=H_{2}(\pi)$. On the other hand, if $X$ is a 0 -connected CW-complex with $\Omega X$ contractible, then $X$ itself is contractible.

1. The model categories $\mathscr{C}$ and $\mathscr{F}$. Let $\mathscr{C}$ denote the category of all modules over a Dedekind domain $\Lambda$. Defining cofibrations, weak equivalences and fibrations to be respectively monomorphisms, $i$-homotopy equivalences in the sense of Hilton [2] and maps satisfying the lifting property (L.P.) below, the author showed in [8] that $\mathscr{C}$ is a model category satisfying the axioms $M_{0}$ to $M_{5}$ of Quillen [6].

(L.P.) A map $p: E \rightarrow B$ in $\mathscr{C}$ satisfies (L.P.) if given any $f: J \rightarrow B$ with $J$ injective there exists a lift $g: J \rightarrow E$ of $f$ (i.e. $p g=f$ ).

Let $\mathscr{F}$ be the category of finitely generated modules over a Principal Ideal Domain (PID). Defining fibrations, weak equivalences and cofibrations to be respectively epimorphisms, $p$-homotopy equivalences in the sense of Hilton [2] and maps satisfying the extension property (E.P.) mentioned below it was shown in [8] that $\mathscr{F}$ is a model category in the sense of Quillen.

(E.P.) A map $q: A \rightarrow E$ is said to have the (E.P.) if given any finitely generated free $\Lambda$-module $F$ and any map $\alpha: A \rightarrow F$ there exists a map $\beta: E \rightarrow F$ satisfying $\beta q=\alpha$.

It is clear that for any $M$ in $\mathscr{C}$ (resp. $\mathscr{F}$ ) $M \times M \rightarrow^{\mu} M, M \rightarrow^{\sigma} M$ defined by $\mu(x, y)=x+y, \sigma(x)=-x$ make $M$ into a group object in $\mathscr{C}$ (resp. $\mathscr{F}$ ) with $\mu$ as the multiplication, $\sigma$ as the inversion and $0: M \rightarrow M$ as the unit. Similarly, $M \rightarrow^{v} M \oplus M$ given by $\nu(x)=(x, x)$ makes $M$ into a cogroup object in $\mathscr{C}$ (resp. $\mathscr{F}$ ) with $\sigma$ as the inversion and $M \rightarrow^{0} M$ as the co-unit. The following were proved in [8].

(1) In $\mathscr{C}$ as well as $\mathscr{F}$ all the objects are fibrant and cofibrant.

(2) An object $M$ of $\mathscr{C}$ (resp. $\mathscr{F}$ ) is contractible if and only if $M$ is injective (respectively free).

(3) For any $M$ in $\mathscr{C}$ as well as $\mathscr{F}$ both $\Sigma M$ and $\Omega M$ are contractible.

(4) For $M$ in $\mathscr{C}$ or $\mathscr{F}$

(a) Ind Cat $M=0=$ Cocat $M$ if and only if $M$ is contractible.

(b) Ind Cat $M=\infty=$ Cocat $M$ whenever $M$ is not contractible.

Proposition 1.1. Let $M \in \mathscr{C}$ (resp. $\mathscr{F})$.

(i) If there exists a fibration $p: E \rightarrow{ }^{p} B$ with $E$ contractible and fibre of $p$ isomorphic to $M$, then $M$ itself is contractible.

(ii) If there exists a cofibration $q: A \rightarrow E$ with $E$ contractible and cofibre of $q$ isomorphic to $M$, then $M$ itself is contractible.

Proof. If there exists a fibration $E \rightarrow{ }^{p} B$ with $E$ contractible and fibre of $p$ isomorphic to $M$ then, from the definition of Cocat $M$, we see 
that Cocat $M \leqq 1$. Then $4(\mathrm{~b})$ implies $M$ is contractible. This proves (i). The proof of (ii) is exactly dual and hence omitted.

2. Contractibility of $\Sigma X$. We now consider the category $\mathscr{T}_{*}$ of pointed topological spaces. Unless otherwise mentioned the homology groups we consider are the singular homology groups.

Proposition 2.1. Let $X$ be a topological space which is of the homotopy type of $\Omega Y$ for some $Y$. Then $\Sigma X$ is contractible if and only if $X$ itself is contractible.

Proof. When $X$ is contractible clearly $\Sigma X$ also is. Assume $\Sigma X$ contractible. Let $f: X \rightarrow \Omega Y$ be a homotopy equivalence. Then

$$
[X, X] \stackrel{f_{*}}{\simeq}[X, \Omega Y] \simeq[\Sigma X, Y]=0
$$

since $\Sigma X$ is contractible. Thus $[X, X]=0$, and $X$ is contractible.

Corollary 2.2. Let $G$ be a topological group. Then $\Sigma G$ is contractible if and only if $G$ itself is.

Proof. It is known that $G$ is of the homotopy type of $\Omega B_{G}$ where $B_{G}$ is a classifying space for $G$.

REMARK 2.3. When $G$ is a group object in $\mathscr{T}_{*}$ the above corollary asserts that $\Sigma G$ is contractible if and only if $G$ itself is. Consider the model categories $\mathscr{C}$ and $\mathscr{F}$ introduced in $\S 1$. All the objects in $\mathscr{C}$ (or $\mathscr{F}$ ) are group objects; for any object $M$ both $\Sigma M$ and $\Omega M$ are contractible. By taking the base ring $\Lambda$ to be the ring $Z$ of integers we see immediately that not all $M$ in $\mathscr{C}$ (resp. $\mathscr{F}$ ) are contractible.

Definition 2.4. Given any group $\pi$ not necessarily abelian we call a space $X$ a "Moore space" of type $(\pi, 1)$; if $X$ is arcwise connected, $\pi_{1}(X) \simeq \pi$ and $H_{j}(X)=0$ for $j \geqq 2$.

This definition differs from the one given in [9] in only one respect. We allow $\pi$ to be nonabelian. We denote a Moore space of type $(\pi, 1)$ by $M(\pi, 1)$. Let $H_{i}(\pi)$ denote the $i$ th homology group of the group $\pi$ with coefficients in $Z$ (with trivial $\pi$-operators). The following is proved in [9].

Proposition 2.5. A Moore space $M(\pi, 1)$ exists if and only if $H_{2}(\pi)=0$.

The proof given in [9] is valid even if $\pi$ is not abelian. When $H_{2}(\pi)=0$ the construction in [9] actually gives an $M(\pi, 1) \mathrm{CW}$-complex.

Proposition 2.6. Let $X$ be a $\mathrm{CW}$-complex. Then $\Sigma X$ is contractible if and only if $X$ is an $M(\pi, 1)$ complex with $H_{1}(\pi)=0=H_{2}(\pi)$.

Proof. Assume $\Sigma X$ contractible. If $\alpha$ is the cardinality of the set of arc components of $X$ then $H_{1}(\Sigma X)$ is free abelian of rank $\alpha-1$. Since 
$H_{1}(\Sigma X)=0$ we see that $\alpha=1$. Thus $X$ is 0 -connected. Let $\pi$ denote $\pi_{1}(X)$. Then from $0=H_{j+1}(\Sigma X) \simeq H_{j}(X)$ for $j \geqq 1$ we see that $H_{1}(X) \simeq \pi /[\pi, \pi] \simeq$ $H_{1}(\pi)=0$ and $H_{j}(X)=0$ for $j \geqq 2$. Hence, $X$ is an $M(\pi, 1)$ complex with $H_{1}(\pi)=0$. From Proposition 2.5 we get $H_{2}(\pi)=0$.

Conversely, assume $X$ is an $M(\pi, 1) \mathrm{CW}$-complex with $H_{1}(\pi)=0$. $\Sigma X$ is simply connected (Van Kampen theorem). From $H_{j+1}(\Sigma X)=H_{j}(X)$ for $j \geqq 1, H_{j}(X)=0$ for $j \geqq 2$ and $H_{1}(X) \simeq \pi /[\pi, \pi] \simeq H_{1}(\pi)=0$ we immediately see that $H_{i}(\Sigma X)=0$ for all $i \geqq 1$. By J. H. C. Whitehead $\Sigma X$ is contractible.

REMARK 2.7. Finitely presentable groups $\pi$ satisfying $H_{1}(\pi)=0=H_{2}(\pi)$ are known to be the groups which occur as the fundamental groups of smooth homology $n$-spheres ( $n \geqq 5)$ [4]. There are many such nontrivial groups.

Thus there are noncontractible $\mathrm{CW}$-complexes $X$ with $\Sigma X$ contractible.

\section{Contractibility of $\Omega X$.}

Lemma 3.1. Suppose $X$ is of the homotopy type of a 0-connected CWcomplex. Then $\Omega X$ is contractible if and only if $X$ is.

This is an immediate consequence of the relation $\pi_{i}(\Omega X) \simeq \pi_{i+1}(X)$ for $i \geqq 0$ and J. H. C. Whitehead's theorem.

Example 3.2. Let $A_{1}, A_{2}, A_{3}, A_{4}$ be the subsets of the plane $R^{2}$ given by $\begin{array}{ll}A_{1}=\left\{\left(x, \sin x^{-1}\right) \mid 0<x \leqq \frac{1}{2} \pi^{-1}\right\}, & A_{2}=\left\{\left(\frac{1}{2} \pi^{-1}, y\right) \mid-2 \leqq y \leqq 0\right\}, \\ A_{3}=\left\{(x,-2) \mid 0 \leqq x \leqq \frac{1}{2} \pi^{-1}\right\}, & A_{4}=\{(0, y) \mid-2 \leqq y \leqq 1\} .\end{array}$

Let $X=A_{1} \cup A_{2} \cup A_{3} \cup A_{4}$. Let $x_{0}=(0,1)$ be chosen as the base point in $X$. It is known that the space $\Omega\left(X, x_{0}\right)$ is contractible. However $X$ is not contractible. In fact, the Cech homology $\breve{H}_{1}(X) \simeq Z$; whereas the singular homology group $H_{1}(X)=0$. Hence, $X$ is not even of the homotopy type of a CW-complex.

Remark 3.3. Suppose $X$ is a 0 -connected noncontractible space with $\Omega(X)$ contractible. From Lemma 3.1 we immediately get that such an $X$ will not be of the homotopy type of a CW-complex.

RemarK 3.4. Let $X$ be the space $A_{1} \cup A_{2} \cup A_{3} \cup A_{4}$ given in Example 3.2. Using the fact that Cech cohomology theory satisfies the axioms of Eilenberg-Steenrod we get, in the usual way as a consequence of the exactness homotopy and excision axioms, $\breve{H}^{i+1}(\Sigma X) \simeq \breve{H}^{i}(X)$ for $i \geqq 1$. In particular, $\breve{H}^{2}(\Sigma X) \simeq \breve{H}^{1}(X) \simeq Z$. Hence, $\Sigma X$ is not contractible. The same argument (repeated) yields that none of the spaces $\Sigma^{l} X(l \geqq 1)$ is contractible.

It might be interesting to find an example of a topological space $X$ such that both $\Sigma X$ and $\Omega X$ are contractible but $X$ itself is not. 


\section{REFERENCES}

1. A. Dold, Partitions of unity in the theory of fibrations, Ann. of Math. (2) 78 (1963), 223-255. MR 27 \#5264.

2. P. J. Hilton, Homotopy theory of modules and duality, Internat. Sympos. on Algebraic Topology, Universidad Nacional Autónoma de México and UNESCO, Mexico City, 1958, pp. 273-281. MR 20 \#4588.

3. - Homotopy theory and duality, Gordon and Breach, New York, 1965. MR 33 \#6624.

4. M. A. Kervaire, Smooth homology spheres and their fundamental groups, Trans. Amer. Math. Soc. 144 (1969), 67-72. MR 40 \#6562.

5. J. W. Milnor, Construction of universal bundles. I, Ann. of Math. (2) 63 (1956), 272-284. MR 17, 994.

6. D. G. Quillen, Homotopical algebra, Lecture Notes in Math., no. 43, SpringerVerlag, Berlin and New York, 1967. MR 36 \#6480.

7. K. Varadarajan, Numerical invariants in homotopical algebra, J. Pure Appl. Algebra (submitted).

8. - Numerical invariants in homotopical algebra. II, Applications, J. Pure Appl. Algebra (submitted).

9. —_ Groups for which Moore spaces $M(\pi, 1)$ exist, Ann. of Math. (2) 84 (1966), pp. 368-371. MR 34 \#2017.

Department of Mathematics, University of Calgary, Calgary, Alberta, CANADA 